PROCEEDINGS OF THE

AMERICAN MATHEMATICAL SOCIETY

Volume 131, Number 6, Pages 1655-1660

S 0002-9939(03)06923-5

Article electronically published on January 15, 2003

\title{
AUTOMORPHISMS OF THE ENDOMORPHISM SEMIGROUP OF A FREE MONOID OR A FREE SEMIGROUP
}

\author{
G. MASHEVITZKY AND BORIS M. SCHEIN
}

(Communicated by Stephen D. Smith)

\begin{abstract}
We determine all isomorphisms between the endomorphism semigroups of free monoids or free semigroups and prove that automorphisms of the endomorphism semigroup of a free monoid or a free semigroup are inner or "mirror inner". In particular, we answer a question of B. I. Plotkin.
\end{abstract}

\section{INTRODUCTION}

One of the venerable algebraic problems, the first instance of which was considered by É. Galois, is (using the words of S. Ułam [7]) "determination of a mathematical structure from a given set of endomorphisms". Let $\operatorname{End}(A)$ and $\operatorname{Aut}(A)$ denote the endomorphism monoid and the automorphism group of an algebraic system $A$, respectively. What can be said about systems $A$ and $B$ if $\operatorname{End}(A)$ is isomorphic to $\operatorname{End}(B)$ ? This problem has been considered by numerous authors.

We consider this problem for $\operatorname{End}\left(X^{*}\right)$ and $\operatorname{End}\left(X^{+}\right)$, where $X^{*}$ and $X^{+}$denote, respectively, the free monoid and the free semigroup generated by a set $X$. This particular problem about $\operatorname{End}(A)$, for $A$ a free algebra in a certain variety, was raised by B. I. Plotkin 2 in his lectures on universal algebraic geometry. An analogous problem for $\operatorname{End}(F)$ with $F$ a free group was solved by Formanek [1]. Other examples are given in a remark at the end of this paper.

Recall that the elements of $X^{*}$ are words over $X$, including the empty word 1 . The elements of $X^{+}$are nonempty words. If $u=x_{i_{1}} \ldots x_{i_{k}} \in X^{*}$, then $\bar{u}$ denotes the "opposite" word $x_{i_{k}} \ldots x_{i_{1}}$. In particular, $\overline{1}=1$. Every bijection $f: X \rightarrow Y$ induces an isomorphism $\iota_{f}: X^{*} \rightarrow Y^{*}$ and an anti-isomorphism $\bar{\iota}_{f}: X^{*} \rightarrow Y^{*}$ defined as follows: $\iota_{f}(u)=f\left(x_{i_{1}}\right) \ldots f\left(x_{i_{k}}\right)$ and $\bar{\iota}_{f}(u)=\overline{\iota_{f}(u)}=f\left(x_{i_{k}}\right) \ldots f\left(x_{i_{1}}\right)$. Analogous facts are true for $X^{+}$and $Y^{+}$.

Let $\iota: S \rightarrow T$ be an isomorphism or an anti-isomorphism of a semigroup $S$ onto a semigroup $T$. Define the mapping $\iota \square \iota: \operatorname{End}(S) \rightarrow \operatorname{End}(T)$ by $\iota \square \iota(\varphi)=\iota \circ \varphi \circ \iota^{-1}$ for all $\varphi \in \operatorname{End}(S)$. Thus, if $\iota\left(s_{1}\right)=t_{1}, \iota\left(s_{2}\right)=t_{2}$, and $\varphi\left(s_{1}\right)=s_{2}$ for some $s_{1}, s_{1} \in S$ and $t_{1}, t_{2} \in T$, then $\iota \square \iota(\varphi)\left(t_{1}\right)=t_{2}$. It is easy to see that $\iota \square \iota$ is an isomorphism of $\operatorname{End}(S)$ onto $\operatorname{End}(T)$. We call it the isomorphism induced by $\iota$.

Let $|X|$ denote the cardinality of $X$ and $\mathbb{P}$ the set of prime numbers. A permutation of a finite or an infinite set is a bijection of that set onto itself.

Received by the editors December 5, 2001.

1991 Mathematics Subject Classification. Primary 20M20, 20M05, 08A35.

Key words and phrases. Free semigroup, free monoid, endomorphism, automorphism. 
Theorem 1. Let $\operatorname{End}\left(X^{*}\right)$ and $\operatorname{End}\left(Y^{*}\right)$ be isomorphic.

If $|X|=1$, then $|Y|=1$ and the isomorphisms of $\operatorname{End}\left(X^{*}\right)$ onto $\operatorname{End}\left(Y^{*}\right)$ are in a natural one-to-one correspondence with permutations of $\mathbb{P}$ (explained in the proof).

If $|X| \geq 1$, then every isomorphism $\alpha: \operatorname{End}\left(X^{*}\right) \rightarrow \operatorname{End}\left(Y^{*}\right)$ is induced either by the isomorphism $\iota_{f}$ or by the anti-isomorphism $\bar{\iota}_{f}$ of $X^{*}$ onto $Y^{*}$ for a uniquely determined bijection $f: X \rightarrow Y$. In other words, either $\alpha=\iota_{f} \square \iota_{f}$ or $\alpha=\bar{\iota}_{f} \square \bar{\iota}_{f}$.

The same results hold for every isomorphism $\alpha: \operatorname{End}\left(X^{+}\right) \rightarrow \operatorname{End}\left(Y^{+}\right)$.

Let $\operatorname{Aut}(X)$ and $C_{2}$ denote the symmetric group on $X$ and a 2-element group, respectively. Also, $\mu$ is the so-called mirror automorphism (see Definition [1.4(iii)).

Theorem 2. The groups $\operatorname{Aut}\left(\operatorname{End}\left(X^{+}\right)\right)$and $\operatorname{Aut}\left(\operatorname{End}\left(X^{*}\right)\right)$ are isomorphic.

If $|X|>1$, every automorphism of $\mathbf{E n d}\left(X^{*}\right)$ and of $\mathbf{E n d}\left(X^{+}\right)$is either inner or a product of an inner automorphism and the mirror automorphism $\mu$. In this case $\operatorname{Aut}\left(\operatorname{End}\left(X^{*}\right)\right)$ is isomorphic to the direct product $\operatorname{Aut}(X) \times C_{2}$.

If $|X|=1, \operatorname{Aut}\left(\operatorname{End}\left(X^{*}\right)\right)$ is isomorphic to the symmetric group on a countably infinite set.

In Theorem 2 an automorphism is inner if it is of the form $\iota_{f}$, where $f$ is a permutation of $X$.

\section{Notations AND PRELiminaries}

We give the proofs in the case of the free monoid $X^{*}$. The proofs in the case of the free semigroup $X^{+}$are almost verbatim the same, so we give only a few remarks in the case of free semigroups. Each endomorphism $\varphi$ of $X^{*}$ and of $X^{+}$is uniquely determined by a mapping $X \rightarrow X^{*}$ or, respectively, $X \rightarrow X^{+}$. To define $\varphi$, it suffices to define $\varphi(x)$ for all $x \in X$. The mapping $\varphi \mapsto \varphi^{*}$ such that $\varphi^{*}(x)=\varphi(x)$ and $\varphi^{*}(1)=1$ defines an injective homomorphism of $\operatorname{End}\left(X^{+}\right)$into $\operatorname{End}\left(X^{*}\right)$. For simplicity we identify $\operatorname{End}\left(X^{+}\right)$with a subsemigroup of $\operatorname{End}\left(X^{*}\right)$.

Clearly, $\varphi$ is an automorphism precisely when its restriction to $X$ is a permutation of $X$. Thus the automorphism groups $\operatorname{Aut}\left(X^{*}\right)$ and $\operatorname{Aut}\left(X^{+}\right)$of $X^{*}$ and $X^{+}$ are isomorphic to the symmetric group $\operatorname{Aut}(X)$ of all permutations of $X$.

Definition 1.1. (i) Let $u=x_{i_{1}} \ldots x_{i_{k}} \in X^{*}$. Denote the length $k$ of $u$ by $|u|$. The empty word 1 has length 0 .

(ii) Let $\mathbf{c}(u)$ be the set of all letters of $u$.

(iii) An endomorphism $\varphi \in \operatorname{End}\left(X^{*}\right)$ is linear if $\varphi(x) \in X \cup\{1\}$ for every $x \in X$. In the case of $\operatorname{End}\left(X^{+}\right), \varphi$ is linear when $\varphi(x) \in X$ for all $x \in X$.

(iv) If $u \in X^{*}$ is a fixed word, let $c_{u}$ be the endomorphism of $X^{+}$such that $c_{u}(x)=u$ for all $x \in X$. We call $c_{u}$ a constant endomorphism. Observe that the range of $c_{u}$ does not consist of a single word, unless $u=1$. Clearly, $c_{1}$ is the zero element of $\operatorname{End}\left(X^{*}\right)$. We denote it by 0 , that is, $c_{1}=0$. If $v \in X^{*}$, then $c_{u}(v)=u^{|v|}$.

(v) An endomorphism $\varphi \in \operatorname{End}\left(X^{*}\right)$ is called full if $\varphi \psi=0 \Rightarrow \psi=0$ for all $\psi \in \operatorname{End}\left(X^{*}\right)$.

Lemma 1.2. (i) $\varphi$ is a constant endomorphism of $\operatorname{End}\left(X^{*}\right)$ if and only if $\varphi \alpha=\varphi$ for all $\alpha \in \operatorname{Aut}\left(X^{*}\right)$. The same holds for $\operatorname{End}\left(X^{+}\right)$;

(ii) $\varphi c_{u}=c_{\varphi(u)}$ for all $\varphi \in \operatorname{End}\left(X^{*}\right)$; 
(iii) $\varphi \in \operatorname{End}\left(X^{*}\right)$ is a constant idempotent if and only if either $\varphi=c_{x}$ for some $x \in X$ or $\varphi=0$;

(iv) $\varphi \in \operatorname{End}\left(X^{*}\right)$ is full if and only if $\varphi(x) \neq 1$ for all $x \in X$.

Proof. (i) If $x \in X$, then $\alpha(x)=y \in X$. Varying $\alpha$, we obtain $c_{u} \alpha(x)=c_{u}(y)=u$ for all $y \in X$. Thus $c_{u} \alpha=c_{u}$. Conversely, let $\varphi \alpha=\varphi$ for all automorphisms $\alpha$. If $\alpha(x)=y$ for some $x, y \in X$, then $\varphi(x)=\varphi \alpha(x)=\varphi(y)$. Varying $\alpha$, we obtain $\varphi(x)=\varphi(y)$ for all $x, y \in X$. It follows that $\varphi=c_{u}$, where $u=\varphi(x)$ for any $x \in X$.

(ii) $\varphi c_{u}(x)=\varphi(u)$ for every $x \in X$. Thus $\varphi c_{u}=c_{\varphi(u)}$.

(iii) If $\varphi$ is a constant idempotent, that is, $\varphi=c_{u}$ for some $u \in X^{*}$ and $c_{u} c_{u}=c_{u}$, then, by (ii), $c_{u}=c_{u} c_{u}=c_{c_{u}(u)}=c_{u|u|}$. Thus $u^{|u|}=u$, that is, $|u| \leq 1$. The converse is obvious.

(iv) If $\varphi(x)=1$ for some $x \in X$, then $c_{x} \neq 0$ but $\varphi c_{x}=0$. Thus $\varphi$ is not full.

Conversely, let $\varphi(x) \neq 1$ for all $x \in X$. Thus $\varphi(u) \neq 1$ for all $u \in X^{+}$. If $\psi \neq 0$, then $\psi(x)=u \neq 1$ for some $x \in X$, and hence $\psi c_{x}=c_{\psi(x)}=c_{u} \neq 0$. Thus $\varphi \psi c_{x}=\varphi c_{u}=c_{\varphi(u)} \neq 0$, so that $\varphi \psi \neq 0$. It follows that $\varphi$ is full.

Lemma 1.3. The mapping $\mu(\varphi)=\bar{\varphi}$ is an automorphism of $\operatorname{End}\left(X^{*}\right)$.

Proof. Obviously, $f: u \rightarrow \bar{u}$ is an antiautomorphism of $X^{*}$ and $f^{-1}=f$. Thus $f^{-1} \varphi f=\bar{\varphi}$, and Lemma 1.3 follows from this equality.

Definition 1.4. (i) Let $\alpha: \operatorname{End}\left(X^{*}\right) \rightarrow \operatorname{End}\left(Y^{*}\right)$ be an isomorphism. By parts (i) and (iii) of Lemma 1.2 for every $x \in X$ there exists $y \in Y$ such that $\alpha\left(c_{x}\right)=c_{y}$. Define a bijection $f: X \rightarrow Y$ by $f(x)=y$. We say that $f$ is induced by $\alpha$. We make an analogous definition for $\operatorname{End}\left(X^{+}\right) \rightarrow \operatorname{End}\left(Y^{+}\right)$.

(ii) An automorphism $\alpha$ of $\operatorname{End}\left(X^{*}\right)$ or of $\operatorname{End}\left(X^{+}\right)$is stable if it induces the identity permutation of $X$, that is, $\alpha\left(c_{x}\right)=c_{x}$ for all $x \in X$.

(iii) The mapping $\mu$ of Lemma 1.3 is the mirror automorphism of $\operatorname{End}\left(X^{*}\right)$.

\section{The CASE $|X|=1$}

If $|X|>1$, let $x$ and $y$ be distinct elements of $X$. Then $c_{x y}\left(c_{x}(x)\right)=c_{x y}(x)=$ $x y \neq x x=c_{x}(x y)=c_{x} c_{x y}(x)$, and hence $c_{x y} c_{x} \neq c_{x} c_{x y}$. Thus $\operatorname{End}\left(X^{*}\right)$ and $\operatorname{End}\left(X^{+}\right)$are not commutative.

If $X=\{x\}$, a singleton, then $X^{+}=\left\{x, x^{2}, x^{3}, \ldots\right\}$ consists of all powers of $x$ and is isomorphic to the additive semigroup $(\mathbb{N},+)$ of positive integers. Every element of $\operatorname{End}\left(X^{+}\right)$corresponds to $\varphi_{k}=\left(\begin{array}{c}x \\ x^{k}\end{array}\right)$ for some $k \in \mathbb{N}$, and $\operatorname{End}\left(X^{+}\right)$is isomorphic to the multiplicative semigroup $(\mathbb{N}, \cdot)$ of positive integers. $(\mathbb{N}, \cdot)$ is a free commutative monoid with the countably infinite set $\mathbb{P}$ of free generators that are prime numbers. Therefore, $\operatorname{End}\left(X^{+}\right)$is a free commutative semigroup and $\left\{\varphi_{k}\right\}_{k \in \mathbb{P}}$ is its set of free generators. If $\alpha: \operatorname{End}\left(X^{+}\right) \rightarrow \operatorname{End}\left(Y^{+}\right)$is an isomorphism, then $\operatorname{End}\left(Y^{+}\right)$ is commutative, and hence $|Y|=1$. Thus $\alpha$ is uniquely determined by a bijection between the free generators of $\operatorname{End}\left(X^{+}\right)$and $\operatorname{End}\left(Y^{+}\right)$. These bijections (and hence the isomorphisms) are in a one-to-one correspondence with permutations of $\mathbb{P}$. The elements of $\operatorname{Aut}\left(\operatorname{End}\left(X^{+}\right)\right)$correspond to permutations of generators, and thus $\operatorname{Aut}\left(\operatorname{End}\left(X^{+}\right)\right)$is an infinite group isomorphic to the symmetric group $\operatorname{Aut}(\mathbb{P})$ of all permutations of $\mathbb{P}$, and also isomorphic to $\operatorname{Aut}\left(\mathbb{P}^{+}\right)$because $\mathbb{P}$ is countably infinite. 
Similarly, $X^{*}$ is isomorphic to the additive semigroup $\left(\mathbb{N}_{0},+\right)$ of nonnegative integers, $\operatorname{End}\left(X^{*}\right)$ is isomorphic to the multiplicative semigroup $\left(\mathbb{N}_{0}, \cdot\right)$ of nonnegative integers, and hence $\operatorname{Aut}\left(\operatorname{End}\left(X^{*}\right)\right)$ is isomorphic to $\operatorname{Aut}\left(\operatorname{End}\left(X^{+}\right)\right)$.

\section{Stable automorphisms of $\operatorname{End}\left(X^{*}\right)$ and $\operatorname{End}\left(X^{+}\right)$}

Lemma 3.1. (i) If $\alpha$ is a stable automorphism of $\operatorname{End}\left(X^{*}\right), \varphi \in \operatorname{End}\left(X^{*}\right)$, and $x \in X$, then $\alpha\left(c_{\varphi(x)}\right)=c_{\alpha(\varphi)(x)}$.

(ii) If $\varphi$ is linear, then $\alpha(\varphi)=\varphi$.

Proof. (i) By Lemma 1.2(ii), $\alpha\left(c_{\varphi(x)}\right)=\alpha\left(\varphi c_{x}\right)=\alpha(\varphi) \alpha\left(c_{x}\right)=\alpha(\varphi) c_{x}=c_{\alpha(\varphi)(x)}$.

(ii) If $\varphi$ is linear, then $\varphi(x) \in X \cup\{1\}$, and hence $c_{\alpha(\varphi)(x)}=\alpha\left(c_{\varphi(x)}\right)=c_{\varphi(x)}$. Therefore, $\alpha(\varphi)(x)=\varphi(x)$ for every $x \in X$, and so $\alpha(\varphi)=\varphi$.

Lemma 3.2. If $\alpha\left(c_{u}\right)=c_{v}$, where $\alpha$ is a stable automorphism of $\operatorname{End}\left(X^{*}\right)$ and $u, v \in X^{*}$, then $\mathbf{c}(u)=\mathbf{c}(v)$.

Proof. If $z \in \mathbf{c}(u) \backslash \mathbf{c}(v)$, choose $x \in X, \varphi \in \operatorname{End}\left(X^{*}\right)$, and $g \in \operatorname{End}\left(X^{+}\right)$such that $x \neq z, \varphi(x)=u, g(z)=x$, and $g(y)=y$ for all $y \neq z, y \in X$. Then $g$ is linear, $g(v)=v, \alpha(g)=g$ by Lemma 3.1 (ii), and $\alpha\left(c_{u}\right)=c_{v}=c_{g(v)}=g c_{v}=\alpha(g) \alpha\left(c_{u}\right)=$ $\alpha\left(g c_{u}\right)=\alpha\left(c_{g(u)}\right)$. By injectivity of $\alpha, c_{u}=c_{g(u)}$, so that $u=g(u)$, which is not true. Thus $\mathbf{c}(u) \backslash \mathbf{c}(v)=\varnothing$. Similarly, $\mathbf{c}(v) \backslash \mathbf{c}(u)=\varnothing$. Therefore, $\mathbf{c}(u)=\mathbf{c}(v)$.

Lemma 3.3. If $\alpha$ is a stable automorphism of $\operatorname{End}\left(X^{*}\right)$, then $|\varphi(x)|=|\alpha(\varphi)(x)|$ for all $\varphi \in \operatorname{End}\left(X^{*}\right)$ and $x \in X$.

Proof. Suppose that $\left|\varphi_{1}(x)\right|=\left|\varphi_{2}(x)\right|=m,\left|\alpha\left(\varphi_{1}\right)(x)\right|=k$, and $\left|\alpha\left(\varphi_{2}\right)(x)\right|=l$. Then $c_{x} \varphi_{1} c_{x}=c_{x} \varphi_{2} c_{x}=c_{x^{m}}$. Also, $\alpha\left(c_{x}\right)=c_{x}$. Therefore, $\alpha\left(c_{x^{m}}\right)=\alpha\left(c_{x} \varphi_{1} c_{x}\right)=$ $c_{x} \alpha\left(\varphi_{1}\right) c_{x}=c_{x^{k}}$ and $\alpha\left(c_{x^{m}}\right)=\alpha\left(c_{x} \varphi_{2} c_{x}\right)=c_{x} \alpha\left(\varphi_{2}\right) c_{x}=c_{x^{l}}$. Thus $k=l$.

If $Y$ is a finite $n$-element subset of $X$ and $m$ a nonnegative integer, define $\operatorname{End}_{Y}^{m}(x)=\left\{\varphi \in \operatorname{End}\left(X^{*}\right)|| \varphi(x) \mid=m\right.$ and $\left.\mathbf{c}(\varphi(x))=Y\right\}$. By the previous paragraph and Lemma 3.2 , for every $m$ there exists $k$ such that $\alpha\left(E n d_{Y}^{m}(x)\right) \subseteq E n d_{Y}^{k}(x)$. There are $n^{k}$ words of length $k$ and $n^{m}$ words of length $m$ over $Y$. Since $\alpha$ is injective, then $k \geq m$. Since $\alpha$ is surjective, $k=m$. Thus $\alpha\left(\operatorname{End}_{Y}^{m}(x)\right)=\operatorname{End} d_{Y}^{m}(x)$, and hence $|\varphi(x)|=|\alpha(\varphi)(x)|$ for all $\varphi \in \operatorname{End}\left(X^{*}\right)$ and $x \in X$.

Corollary 3.4. Choose two distinct elements $x_{1}, x_{2} \in X$. If $\alpha$ is a stable automorphism of $\operatorname{End}\left(X^{*}\right)$, then $\alpha\left(c_{x_{1} x_{2}}\right)$ is either $c_{x_{1} x_{2}}$ or $c_{x_{2} x_{1}}$.

Proof. By Lemma 1.2(i), $\alpha\left(c_{x_{1} x_{2}}\right)=c_{u}$ for some $u \in X^{*}$. By Lemma 3.2, $\mathbf{c}(u)=$ $\left\{x_{1}, x_{2}\right\}$. By Lemma 3.3. $|u|=2$. Thus $u$ is either $x_{1} x_{2}$ or $x_{2} x_{1}$.

Lemma 3.5. If $\alpha$ is a stable automorphism of $\operatorname{End}\left(X^{*}\right)$ and $\alpha\left(c_{x_{1} x_{2}}\right)=c_{x_{1} x_{2}}$ for some $x_{1}, x_{2} \in X, x_{1} \neq x_{2}$, then $\alpha$ is the identity automorphism of $\operatorname{End}\left(X^{*}\right)$.

Proof. First we prove that $\alpha\left(c_{u}\right)=c_{u}$ for all $u \in X^{*}$ by induction on $|u|$. The base of induction is obvious for $|u|=0$ and follows from the stability of $\alpha$ for $|u|=1$. Let the claim hold for $|u|<k$, and let $|u|=k$, where $u=u_{1} y$. If $\varphi\left(x_{1}\right)=u_{1}, \varphi\left(x_{2}\right)=y$, and $\varphi(x)=x$ for other $x \in X$, then $\varphi c_{x_{1} x_{2}}=c_{u}$. By the induction hypothesis, $\alpha\left(c_{u_{1}}\right)=c_{u_{1}}$. Also, $\alpha\left(c_{x}\right)=c_{x}$ for every $x \in X$. By Lemma 3.1 (i), $\alpha(\varphi)(x)=\varphi(x)$ for all $x \in X$. Thus $\alpha(\varphi)=\varphi$, and hence $\alpha\left(c_{u}\right)=\alpha\left(\varphi c_{x_{1} x_{2}}\right)=\varphi c_{x_{1} x_{2}}=c_{u}$.

By Lemma 3.1(i), $c_{\varphi(x)}=\alpha\left(c_{\varphi(x)}\right)=c_{\alpha(\varphi)(x)}$, and so $\varphi(x)=\alpha(\varphi(x))$ for all $x \in X$. Thus $\varphi=\alpha(\varphi)$ for all $\varphi \in \operatorname{End}\left(X^{*}\right)$. 
Lemma 3.6. If $\alpha$ is a stable automorphism of $\operatorname{End}\left(X^{*}\right)$ and $\alpha\left(c_{x_{1} x_{2}}\right)=c_{x_{2} x_{1}}$ for some $x_{1}, x_{2} \in X, x_{1} \neq x_{2}$, then $\alpha=\mu$.

Proof. First we prove that $\alpha\left(c_{u}\right)=c_{\bar{u}}$ for all $u \in X^{*}$ by induction on $|u|$. The base of induction is obvious for $|u|=0$ and follows from the stability of $\alpha$ for $|u|=1$. Let the claim hold for $|u|<k$, and let $|u|=k$, where $u=u_{1} y$. If $\varphi\left(x_{1}\right)=u_{1}, \varphi\left(x_{2}\right)=y$, and $\varphi(x)=x$ for other $x \in X$, then $\varphi c_{x_{1} x_{2}}=c_{u}$. By the induction hypothesis, $\alpha\left(c_{u_{1}}\right)=c_{\bar{u}_{1}}$. Also, $\alpha\left(c_{x}\right)=c_{x}$ for every $x \in X$. By Lemma $3.1(\mathrm{i}), \alpha(\varphi)(x)=\overline{\varphi(x)}$ for all $x \in X$. Thus $\alpha(\varphi)=\bar{\varphi}$, and hence $\alpha\left(c_{u}\right)=\alpha\left(\varphi c_{x_{1} x_{2}}\right)=\bar{\varphi} c_{x_{2} x_{1}}=c_{y \bar{u}_{1}}=c_{\bar{u}}$.

By Lemma 3.1 (i), $\alpha(\varphi)=\bar{\varphi}$ for all $\varphi \in \operatorname{End}\left(X^{*}\right)$. Thus $\alpha=\mu$.

We skip the proof of the following proposition (it is obtained as an obvious simplification of our proof of Theorem 2).

Proposition 3.7. Let $F(X)$ be the free commutative monoid with a set $X,|X|>1$, of free generators. Every automorphism of $\operatorname{End}(F(X))$ is inner.

Remark 3.8. Similarly, automorphisms of a free commutative semigroup are inner.

\section{Group of automorphisms of $\operatorname{End}\left(X^{*}\right)$}

Proof of Theorem 1 For $|X|=1$ Theorem 11 was proved in Section 2, Suppose that $|X|>1, \alpha: \operatorname{End}\left(X^{*}\right) \rightarrow \operatorname{End}\left(Y^{*}\right)$ is an isomorphism, and $f: X \rightarrow Y$ is a bijection induced by $\alpha$, that is, $\alpha\left(c_{x}\right)=c_{f(x)}$ for every $x \in X$. Then $f$ extends to an isomorphism $\iota_{f}: X^{*} \rightarrow Y^{*}$ and $\iota_{f}$ induces an isomorphism $\alpha_{f}=\iota_{f} \square \iota_{f}$ : $\operatorname{End}\left(X^{*}\right) \rightarrow \operatorname{End}\left(Y^{*}\right)$. Consider the automorphism $\beta=\alpha_{f}^{-1} \alpha$ of $\operatorname{End}\left(X^{*}\right)$. Then $\beta\left(c_{x}\right)=\alpha_{f}^{-1}\left(\alpha\left(c_{x}\right)\right)=\alpha_{f}^{-1}\left(c_{f(x)}\right)=c_{f^{-1}(f(x))}=c_{x}$ for every $x \in X$ and $\beta$ is stable. By Corollary 3.4 and Lemmas 3.5 and 3.6 is an identity automorphism $\Delta$ or a mirror automorphism $\mu$. In the former case, $\alpha_{f}^{-1} \alpha=\Delta$, and hence $\alpha=\alpha_{f}$, the automorphism induced by $\iota_{f}$. In the latter case, $\alpha_{f}^{-1} \alpha=\mu$, whence $\alpha=\alpha_{f} \mu$, the automorphism induced by $\bar{\iota}_{f}$.

Proof of Theorem Q If $X=Y$, Theorem 1 becomes the first part of Theorem 2 Thus we prove here the second part of Theorem 2 for $|X|>1$.

Obviously, $\{\Delta, \mu\}$ is a two-element group of automorphisms of $\operatorname{End}\left(X^{*}\right)$. It is isomorphic to $C_{2}$. Define a mapping $i: \operatorname{Aut}\left(\operatorname{End}\left(X^{*}\right)\right) \rightarrow \operatorname{Aut}(X) \times C_{2}$ as follows: if $\alpha \in \operatorname{Aut}\left(\operatorname{End}\left(X^{*}\right)\right)$, then $i(\alpha)=(f, \Delta)$ for $\alpha=\alpha_{f}$, and $i(\alpha)=(f, \mu)$ for $\alpha=\alpha_{f} \mu$. Clearly, $i$ is a bijection. Also, $i$ is a homomorphism because $\alpha_{g} \circ \alpha_{f}=\alpha_{g \circ f}$ and $\mu \circ \alpha=\alpha \circ \mu$ for all $f, g \in \operatorname{Aut}(X)$ and $\alpha \in \operatorname{Aut}\left(\operatorname{End}\left(X^{*}\right)\right)$. Thus $i$ is an isomorphism of $\operatorname{Aut}\left(\operatorname{End}\left(X^{*}\right)\right)$ onto $\operatorname{Aut}(X) \times C_{2}$.

Remark 4.1. Let $\mathbf{L}$ be the variety of all left zero semigroups (that is, the semigroups satisfying the identity $x_{1} x_{2}=x_{1}$ ). It is easily seen that every semigroup in this variety is free. If $X$ is a left zero semigroup, then every transformation $X \rightarrow X$ is an endomorphism of $X$, and hence $\operatorname{End}(X)$ is merely the full transformation semigroup $\mathcal{T}_{X}$ of the set $X$. Then $\operatorname{Aut}(\operatorname{End}(X))=\operatorname{Aut}\left(\mathcal{T}_{X}\right)$. Schreier [5] proved that all automorphisms of $\mathcal{T}_{X}$ are inner and $\operatorname{Aut}\left(\mathcal{T}_{X}\right)$ is isomorphic to $\operatorname{Aut}(X)$, the symmetric group on $X$.

Let $\mathbf{Z}$ be the variety of all zero semigroups (that is, the semigroups satisfying the identity $x_{1} x_{2}=x_{3} x_{4}$ ). They are semigroups $X^{0}=X \cup\{0\}, 0 \notin X$, with zero 0 such that $x y=0$ for all $x, y \in X^{0}$. Every semigroup in this variety is free. Every partial transformation $f$ of $X$ corresponds bijectively to an endomorphism $\bar{f}$ of $X^{0}$ : 
for every $s \in X^{0}, \bar{f}(s)=f(s)$ if $f(s)$ is defined, and $\bar{f}(s)=0$ otherwise. Clearly, $\mathfrak{F}_{X}$, the semigroup of all partial transformations of $X$, is naturally isomorphic to $\operatorname{End}\left(X^{0}\right)$ under the correspondence $f \mapsto \bar{f}$, and hence $\operatorname{Aut}(\operatorname{End}(X))$ is isomorphic to $\operatorname{Aut}\left(\mathfrak{F}_{X}\right)$. As proved by Shutov [6], all automorphisms of $\mathfrak{F}_{X}$ are inner and $\operatorname{Aut}\left(\mathfrak{F}_{X}\right)$ is isomorphic to $\operatorname{Aut}(X)$, the symmetric group on $X$.

\section{REFERENCES}

1. E. Formanek, A question of B. Plotkin about the semigroup of endomorphisms of a free group, Proc. American Math. Society 130(2001), 935-937. MR 2002h:20032

2. B. I. Plotkin, Seven Lectures on the Universal Algebraic Geometry, Preprint, Institute of Mathematics, Hebrew University, 2000.

3. Boris M. Schein and Beimnet Teclezghi, Endomorphisms of finite full transformation semigroups, Proc. American Math. Soc. 126(1998), 2579-2587. MR 99b:20093

4. Б. М. Шайн, Атомные полугруды и инволютированные полугруппы, Известия Высш. Учебн. Завед., Математика, 1965, №3, 172-184. In Russian: B. M. Schein, Atomic semigroups and involuted semigroups, Izvestiya Vyssh. Uchebn. Zaved. (Matematika), 1965, no. $3,172-184$.

5. J. Schreier, Über Abbildungen einer abstrakten Menge auf ihre Teilmengen, Fundamenta Mathematica 28(1936), 261-264.

6. Э. Г. Шутов, Гомоморфизмы полугруппь всех частичных преобразований, Известия Высш. Учебн. Завед., Математика, 1961, №3, 178-184. In Russian: Ѐ. G. Shutov, Homomorphisms of the semigroup of all partial transformations, Izvestiya Vyssh. Uchebn. Zaved. (Matematika), 1961, no. 3, 178-184.

7. S. M. Ulam, A Collection of Mathematical Problems, Interscience Publishers, New York, 1960. MR 22:10884

Department of Mathematics, Ben Gurion University of the Negev, Beer Sheva, Israel E-mail address: gmash@math.bgu.ac.il

Department of Mathematical Sciences, University of Arkansas, Fayetteville, ARKANSAS 72701

E-mail address: bschein@uark.edu 